\title{
Novel insights into lung regenerative medicine
}

\author{
Toyofumi F. Chen-Yoshikawa, MD, and Ryo Okabe, MD
}

\author{
From the Department of Thoracic Surgery, Kyoto University Graduate School of Medicine, Kyoto, Japan. \\ Disclosures: Authors have nothing to disclose with regard to commercial support. \\ Received for publication Sept 21, 2018; accepted for publication Sept 21, 2018; available ahead of print Oct 29, \\ 2018. \\ Address for reprints: Toyofumi F. Chen-Yoshikawa, MD, Department of Thoracic Surgery, Kyoto University \\ Graduate School of Medicine, 54 Shogoin-Kawahara-cho, Sakyo-ku, 606-8507, Kyoto, Japan (E-mail: \\ fengshic@kuhp.kyoto-u.ac.jp). \\ J Thorac Cardiovasc Surg 2019;157:421-2 \\ $0022-5223 / \$ 36.00$ \\ Copyright (c) 2018 by The American Association for Thoracic Surgery \\ https://doi.org/10.1016/j.jtcvs.2018.09.062
}

Guenthart and colleagues ${ }^{1}$ presented a commendable perspective article providing novel insights into and therapeutic targets for lung regeneration.

Respiratory stem cell research has progressed significantly in the past decade. Guenthart and colleagues ${ }^{1}$ reported the presence of adequate evidence for lung progenitors and the cellular composition of the lung. Type 2 alveolar cells, club cells, and so forth are considered bronchioalveolar stem cells. ${ }^{2}$ Furthermore, the stem cell niche has received increasing attention. Various types of mesenchymal cells facilitate bronchioalveolar cell regeneration from type 2 alveolar cells and club cells in the injured lung. ${ }^{3}$ Furthermore, Guenthart and colleagues ${ }^{1}$ reported a new cell type ${ }^{4}$ (the pulmonary ionocyte). Numerous studies have focused on cystic fibrosis because it is a genetic disorder with numerous reported genetic and molecular mechanisms, for example, pulmonary ionocytes with cystic fibrosis transmembrane conductance regulators. These cells are rare, representing $1 \%$ to $2 \%$ of airway epithelial cells; however, this new cell-targeted therapy for patients with cystic fibrosis might be a unique strategy and is expected to regenerate lung tissue and maintain respiratory function of the injured lung.

Remarkable advancements in lung organoid medicine have been reported since Takahashi and colleagues ${ }^{5}$ reported that induced pluripotent stem (iPS) cells can be induced from adult human fibroblasts in 2007. Yamamoto and colleagues ${ }^{6}$ recently reported efficient methods for long-term expansion of alveolar stem cells derived from human iPS cells in organoids in 2017. Furthermore, Chen and colleagues ${ }^{7}$ successfully organized lung organ organoids from human iPS cells. There are many issues in actualizing cell therapy for acute and chronic lung diseases and performing human clinical trials, thus necessitating further studies.

Furthermore, advancements in lung bioengineering technology have been reported. Bioengineering technology is important to generate cell scaffolds and reconstruct complex lung branches and vasculature. Zhou and colleagues ${ }^{8}$ successfully resisted physiologic blood flow resulting

\section{References} 3025-36.

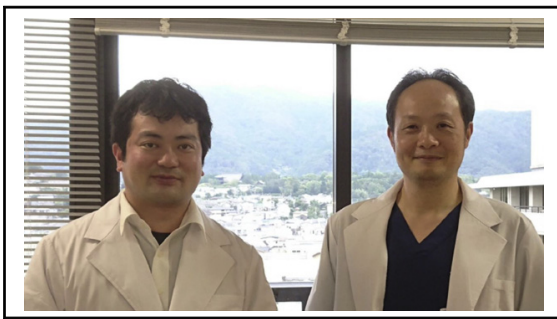

Ryo Okabe, MD, and Toyofumi F. Chen-Yoshikawa MD

\section{Central Message}

Respiratory stem cell research, lung organoid medicine, and lung bioengineering technology have progressed markedly; iPS cells have been induced from adult human fibroblasts.

See Article page 416

from the recipient's pulmonary function and exchanged gases upon ventilation during the 1-hour observation period after lung transplantation with decellularized porcine lung scaffolds and endothelial and epithelial cells. Nichols and colleagues $^{9}$ produced and transplanted a bioengineered lung without generating a pulmonary artery anastomosis in a porcine model without any indication of transplant rejection for 2 months post-transplantation. Studies on lung progenitors and cellular composition are crucial for progress in tissue bioengineering.

Issues including donor shortage and development of new therapies for patients with irreversible respiratory failure warrant resolution. Future studies on cell-targeted therapies, cell therapy, and transplantation therapy for lung regenerative medicine will be required to proceed in an interdisciplinary manner, as reported by Takebe and colleagues, ${ }^{10}$ who reported the need for cooperation with major therapeutic targets in organoid medicine.

1. Guenthart BA, Chen Y-W, Bacchetta M. New insights and therapeutic targets: lung injury and disease. J Thorac Cardiovasc Surg. 2019;157:416-20.

2. Barkauskas CE, Cronce MJ, Rackley CR, Bowie EJ, Keene DR, Stripp BR, et al. Type 2 alveolar are stem cells in adult lung. J Clin Invest. 2013;123:

3. Lee JH, Tammela T, Hofree M, Choi J, Marjanovic ND, Han S, et al. Anatomically and functionally distinct lung mesenchymal populations marked by Lgr5 and Lgr6. Cell. 2017;170:1149-63.

4. Plasschaert LW, Zilionis R, Choo-Wing R, Savova V, Knehr J, Roma G, et al. A single-cell atlas of the airway epithelium reveals the CFTR-rich pulmonary ionocyte. Nature. 2018;560:377-81. 
5. Takahashi K, Tanabe K, Ohnuki M, Narita M, Ichisaka T, Tomoda K, et al. Induction of pluripotent stem cells from human fibroblasts be defined factors. Cell. 2007;131:861-72.

6. Yamamoto Y, Gotoh S, Korogi Y, Seki M, Konishi S, Ikeo S, et al. Long-term expansion of alveolar stem cells derived from human iPS cells in organoids. Nat Methods. 2017:14:1097-106.

7. Chen YW, Huang SX, de Carvalho ALRT, Ho SH, Islam MN, Volpi S, et al. A three-dimensional model of human lung development and disease from pluripotent stem cells. Nat Cell Biol. 2017;19:542-9.
8. Zhou H, Kitano K, Ren X, Rajab TK, Wu M, Gilpin SE, et al. Bioengineering human lung grafts porcine matrix. Ann Surg. 2018; 267:590-8.

9. Nichols JE, La Francesca S, Niles JA, Vega SP, Argueta LB, Frank L, et al. Production and transplantation of bioengineered lung a large-animal model. Sci Transl Med. 2018;10.

10. Takebe T, Wells JM, Helmrath MA, Zom AM. Organoid center strategies for acceleration clinical translation. Cell Stem Cell. 2018;22: 806-9. 